\title{
Effets des niveaux de nutrition en phosphore et en azote et de la composition botanique de communautés prairiales sur l'accumulation de biomasse au printemps
}

\author{
M Duru *, I Calvière \\ Station d'agronomie, Inra, BP 27, F-31326 Castanet-Tolosan, France
}

(Reçu le 28 septembre 1995 ; accepté le 10 avril 1996)

\begin{abstract}
Résumé - L'objectif de cette étude est de hiérarchiser les principaux facteurs de variation de la biomasse aérienne (MS) de communautés prairiales au printemps : composition botanique (CB), niveaux de nutrition en azote (IN) et en phosphore (IP), sachant que ces trois caractéristiques ne sont pas toujours liées du fait notamment de différences de régimes de coupe appliqués aux prairies. Dans les Pyrénées centrales, une communauté végétale témoin à base de graminées et dix-sept autres communautés prairiales bien différenciées par leur niveau de nutrition minérale (en $\mathrm{P}$ et en $\mathrm{N}$ ) et leur composition botanique sont étudiées. Nous observons que la vitesse d'accumulation de la biomasse est réduite de plus de $50 \%$ suite à une réduction du niveau de nutrition azotée (passage d'un niveau de nutrition non limitant IN = 100 à très limitant ( $I N=60)$, de près de $30 \%$ suite à un changement de composition botanique (passage d'espèces caractéristiques d'habitats fertiles à des espèces caractéristiques d'habitats peu fertiles) et d'environ $12 \%$ suite à une réduction du niveau de nutrition en phosphore (passage d'un niveau non limitant à très déficient). Après avoir discuté de l'effet de ces caractéristiques de la prairie en fonction des données de la littérature, nous montrons comment varie, à l'échelle de plusieurs années, la biomasse aérienne d'une prairie composée d'espèces caractéristiques d'habitats peu fertiles suite à un apport de fertilisant : dans un premier temps la biomasse augmente sans que les espèces dominantes aient le temps de changer, puis, au bout de quelques années, la biomasse est encore plus élevée en relation avec le changement des espèces.
\end{abstract}

prairie / température / azote / phosphore / composition botanique

Summary - Effects of phosphorus and nitrogen nutrition status and of botanical composition of permanent pastures on their growth in spring. The objective of this study was to assess the effect of the botanical composition $(B C)$, the nitrogen (IN) and phosphorus (IP) nutrition status on above-ground dry matter accumulation (DM) of permanent pastures in spring; these three factors are not always linked, particularly in relation to the cutting regime. In the central Pyrenees, one plant community dominated by grasses and used as reference and 17 other plant communities well differentiated by their $P$ and $N$ nutrient status and their botanical composition were studied. We observed that the above-ground dry matter accumulation rate decreased by over $50 \%$ when the IN dropped from 100 (optimal $\mathrm{N}$ nutrition) to 60 (low $\mathrm{N}$ available), by nearly $30 \%$ when species characteristic of nutrient-rich habitats were replaced with species characteristic of nutrient-poor habitats, and by about $10 \%$ when the IP dropped from 80 (optimal

\footnotetext{
* Correspondance et tirés à part
} 
$P$ nutrition) to 40 (very low $P$ available). We discuss this data with reference to the literature. Then we show how the above-ground dry-matter changed over the years with the application of fertilizer to a meadow dominated by species that are characteristic of nutrient-poor habitats. At the beginning the above-ground dry-matter increases with no change in botanical composition. Then, after a few years, it keeps increasing as the original species are replaced by more productive ones.

nitrogen / phosphorus / botanical composition

\section{INTRODUCTION}

La biomasse récoltable de communautés prairiales est souvent très variable, même en l'absence de différences de caractéristiques permanentes du milieu (profondeur et texture du sol...). Ces différences de biomasse peuvent provenir des disponibilités en éléments minéraux majeurs (azote, phosphore et potassium), mais aussi des espèces dominantes composant la prairie (Duru et al, 1994). Généralement, les espèces ayant les vitesses relatives de croissance les plus élevées sont associées aux milieux les plus fertiles et réciproquement (Poorter et al, 1990). Toutefois, la connaissance de l'une ou l'autre de ces caractéristiques est insuffisante pour prédire la biomasse récoltable dans la mesure où elles ne sont pas toujours liées (Balent et al, 1993). En effet, des fluctuations de durée limitée quant aux disponibilités en éléments minéraux peuvent se traduire par des différences de biomasse sans pour autant conduire à des changements de composition botanique. De plus, des compositions botaniques différentes peuvent être observées pour un même niveau de nutrition minérale en relation avec la fréquence de coupe et l'intensité de pâturage (Kays et Harper, 1974). Ces raisons font qu'il est nécessaire d'estimer séparément les effets de la composition botanique et des niveaux de nutrition minérale en éléments majeurs sur la biomasse récoltable, soit pour prédire les récoltes, soit pour estimer les conséquences d'un changement des pratiques de fertilisation. À cet effet, on doit disposer de communautés prairiales recouvrant un large gradient de compositions botaniques et de disponibilités en éléments minéraux, soit en échantillonnant des prairies in situ, soit en créant une variabilité par des fertilisations appropriées sur une même prairie.

En l'absence de déficit hydrique, l'azote est le facteur ayant l'effet le plus important sur la croissance. Cependant, les réductions de biomasse dues à la nature des espèces présentes, ainsi qu'à une déficience en d'autres éléments majeurs que l'azote, sont mal connues. La comparaison de la croissance aérienne des espèces prairiales est le plus souvent étudiée en pot, hors compétition pour la lumière (Grime et Hunt, 1975), ce qui rend difficile les transpositions au champ en peuplement dense. Les études de communautés prairiales in situ permettent d'estimer la biomasse récoltable d'un ensemble d'espèces en fonction des états nutritionnels qui leur sont associés, mais les résultats sont alors spécifiques à un mode d'exploitation donné (Fleury, 1994). L'objectif de cette étude est donc de hiérarchiser les effets de chacun des groupes de facteurs (composition botanique, états nutritionnels en azote et en phosphore) sur la croissance aérienne au printemps. Ceci suppose d'une part d'être à même de caractériser ces facteurs, et d'autre part de comparer leurs effets, ce que nous ferons en utilisant un modèle de croissance.

Afin de distinguer et de classer les communautés prairiales d'après les disponibilités en éléments minéraux et leur composition botanique, nous avons fait le choix de considérer une communauté végétale témoin se rapprochant de situations connues (communauté à base de graminées et sans autre déficience en éléments majeurs que l'azote), les autres communautés étant comparées à ce témoin. Pour contourner les difficultés d'estimation des disponibilités en éléments minéraux à partir d'analyses de terre, tout particulièrement dans le cas des prairies permanentes du fait des apports de fertilisants en surface, nous avons préféré estimer a posteriori les niveaux de nutrition minérale de la prairie à partir des teneurs en éléments minéraux du couvert, comme l'ont proposé de nombreux auteurs (Salette, 1982 par exemple).

La modélisation de la croissance aérienne a été le plus souvent réalisée pour des couverts monospécifiques de graminées ou de légumineuses et seulement en fonction des disponibilités en azote. Le modèle le plus fréquemment retenu met en relation la biomasse aérienne avec la quantité de rayonnement intercepté (Monteith, 1972 ; Gosse et al, 1986). L'effet d'un facteur comme l'azote est alors estimé sur la quantité de rayonnement intercepté et sur l'efficience de conversion du rayonnement en biomasse aérien- 
ne. Dans le cas des prairies permanentes, l'utilisation d'un tel modèle se heurte à la difficulté de mesurer la surface foliaire et le rayonnement intercepté, du fait de l'insertion basse et du port des feuilles en fin d'hiver et au début du printemps. On a donc utilisé un modèle de croissance en fonction de la température, certes plus empirique, mais que certains auteurs ont utilisé au printemps (Lemaire, 1985 ; Menzi et al, 1991). Ce choix nous semble acceptable pour deux raisons. Selon Gillet et al (1984), la température conditionne la demande en assimilats alors que l'offre dépend du rayonnement. Or au printemps, ce sont le plus souvent les températures qui sont limitantes. D'autre part, notre objectif est plus de hiérarchiser les effets des caractéristiques de la communauté prairiale que de quantifier précisément des variations de biomasse en fonction du climat.

\section{MATÉRIEL ET MÉTHODES}

\section{Site et dispositifs}

Dans les Pyrénées centrales, trois prairies situées entre 1250 et $1300 \mathrm{~m}$ d'altitude (latitude $47^{\circ} 30^{\prime}$, longitude $2^{\circ}$ ) et distantes de moins de $300 \mathrm{~m}$ ont été échantillonnées en fonction de leur histoire culturale qui, dans le milieu étudié, est corrélée à la disponibilité du sol en éléments minéraux (Balent et Duru, 1984). Ces prairies (S, A, B) sont quasiment plates car situées en terrasses. Lors de la mise en place du dispositif en 1986, elles étaient très différentes du fait de leur composition botanique et des disponibilités en phosphore (teneur du sol en phosphore mesuré par la méthode Dyer et l'état nutritionnel de la prairie : $S$ > $A>B$ ) (Duru, 1992a). Ces prairies sont pâturées par des ovins de début novembre à début mai puis fauchées deux fois ( $S$ et $A$ ) ou une fois (B) en été. Jusqu'au début de chaque expérience, les prairies $S$ et $A$ ont reçu annuellement une fumure organique d'environ $20 \mathrm{t}$ par hectare et par an.

Le premier dispositif a comme fonction d'étudier la croissance en l'absence de déficience en phosphore pour une prairie dont la composition botanique se rapproche de celle d'une prairie de graminées semées. II correspond à la prairie S qui a été étudiée de 1986 à 1988. Chaque automne, à des emplacements différents, une surface de $16 \mathrm{~m}^{2}$ est mise en défens. Dans la première décade de mars, période à partir de laquelle les risques d'enneigement durable au sol sont limités (Charpenteau et Duru, 1983), $120 \mathrm{~kg} \mathrm{ha}^{-1} \mathrm{de}$ $\mathrm{P}_{2} \mathrm{O}_{5}$ et $250 \mathrm{~kg} \mathrm{ha}^{-1}$ de $\mathrm{K}_{2} \mathrm{O}$ sont apportés et deux traitements azotés, 0 et $120 \mathrm{~kg} \mathrm{ha}^{-1}$ (notés respectivement N0 et N1), sont appliqués chacun sur $8 \mathrm{~m}^{2}$. Les apports d'engrais $\mathrm{P}$ et $\mathrm{K}$ visent à compenser les expor- tations de façon à ce que ces éléments ne soient pas limitants pour la croissance.

Le deuxième dispositif a comme fonction d'étudier la croissance pour des prairies très différentes quant à leur composition botanique ainsi qu'à la disponibilité en azote et en phosphore. À cet effet, il combine deux prairies très différentes quant à leur composition botanique lors de la mise en place de l'étude et plusieurs traitements de fertilisation tel que détaillé ci-après. II correspond aux prairies A et B, étudiées en 1991 et 1992. En 1986, trois placettes de $32 \mathrm{~m}^{2}$ ont été délimitées par prairie. Deux d'entre elles reçoivent un apport de phosphore chaque printemps à la mi-mars, de 1986 à 1992 (traitement P2), ou seulement en 1991 et en 1992 (P1). Aucun d'apport de phosphore n'est réalisé sur la troisième placette (traitement $\mathrm{PO}$ ). Un apport d'azote est effectué ( $\mathrm{N} 1: 120 \mathrm{~kg} \mathrm{ha}^{-1}$ ) ou non (NO) en 1991 et 1992 durant la première décade de mars sur des sous placettes de $8 \mathrm{~m}^{2}$ délimitées au sein des placettes de $32 \mathrm{~m}^{2}$. Un apport de potassium $(250 \mathrm{~kg}$ $\mathrm{ha}^{-1}$ ) est réalisé sur l'ensemble du dispositif de façon à largement compenser les exportations en cet élément (Duru, 1992a). Les apports de fertilisants étant susceptibles de modifier la composition botanique, on parlera de communauté végétale pour désigner chaque sous-placette homogène du point de vue des apports d'engrais. Une communauté sera notée $A(B)-P N j$, i et $j$ indiquant les modalités d'apport de phosphore et d'azote selon la codification précisée ci-dessus. Seulement 17 des 24 cas de figure possibles (deux prairies, deux niveaux de fertilisation en azote et trois modalités de fertilisation phosphatée, deux années) ont été étudiées (tableau I).

Calculées sur une base décadaire, les températures moyennes journalières ont été de 3 à $4{ }^{\circ} \mathrm{C}$ au début du mois de mars et ont augmenté jusqu'à $14-15^{\circ} \mathrm{C}$ à la fin du mois de juin. Les corrélations entre les températures moyennes et le rayonnement journalier sont de $0,91,0,82,0,85,0,74,0,80$ respectivement en 1986, 1987, 1988, 1991 et 1992.

\section{Mesures}

La composition botanique a été effectuée à la mi-juin par la méthode de la baïonnette (Daget et Poissonnet, 1971) à partir de 50 points d'observations. Le nombre de contacts totaux de chaque espèce par point d'observation a été noté.

Les prélèvements d'herbe ont été réalisés toutes les deux semaines environ de début avril à la mi-juillet, de façon à établir des courbes d'accumulation de biomasse. Seules les mesures antérieures à la fin juin, période correspondant à la floraison de la plupart des espèces, sont considérées ici, de façon à ne prendre en compte que des végétations où la quantité de matériel sénescent est faible (Duru et al, 1994). Les prélèvements ont été effectués avec des minitondeuses sur des quadrats de $0,25 \mathrm{~m}^{2}$ avec quatre répétitions réparties au hasard au sein de chaque sous-placette. La coupe a été réalisée à $2 \mathrm{~cm}$ de hauteur. Après séchage durant 48 heures à $80^{\circ} \mathrm{C}$, les échan- 
Tableau I. Indices de niveau de nutrition azotée (IN) et phosphatée (IP), classes de composition botanique (1 et 2 respectivement pour les prairies composées d'espèces caractéristiques d'habitats peu ou bien fertiles) pour les différentes communautés végétales.

\begin{tabular}{|c|c|c|c|c|c|c|c|c|c|c|}
\hline \multirow[t]{2}{*}{ Communautés } & \multicolumn{5}{|c|}{1991} & \multicolumn{5}{|c|}{1992} \\
\hline & \multicolumn{2}{|c|}{ IN } & \multicolumn{2}{|c|}{$I P$} & $\begin{array}{c}\text { Composition } \\
\text { botanique }\end{array}$ & \multicolumn{2}{|c|}{ IN } & \multicolumn{2}{|c|}{$I P$} & $\begin{array}{c}\text { Composition } \\
\text { botanique }\end{array}$ \\
\hline A-PONO & 77 & (11) & 55 & (6) & 2 & 75 & (12) & 51 & (4) & 2 \\
\hline A-PON1 & 81 & (8) & 57 & (3) & 2 & 87 & (18) & 47 & (3) & 2 \\
\hline$A-P 2 N 0$ & 86 & (18) & 104 & (7) & 2 & 73 & (9) & 105 & (8) & 2 \\
\hline$A-P 2 N 1$ & 102 & $(7)$ & 97 & (6) & 2 & & & & & \\
\hline B-PONO & 63 & (2) & 49 & (3) & 1 & 71 & (7) & 48 & (3) & 1 \\
\hline B-PON1 & 86 & (5) & 42 & (1) & 1 & 78 & (7) & 36 & (1) & 1 \\
\hline B-P1NO & 80 & (7) & 65 & (2) & 1 & 73 & (7) & 90 & (5) & 1 \\
\hline $\mathrm{B}-\mathrm{P} 1 \mathrm{~N} 1$ & & & & & & 95 & (13) & 84 & (8) & 1 \\
\hline $\mathrm{B}-\mathrm{P} 2 \mathrm{NO}$ & 95 & (8) & 104 & (8) & 2 & 78 & (9) & 108 & (4) & 2 \\
\hline $\mathrm{B}-\mathrm{P} 2 \mathrm{~N} 1$ & 102 & (20) & 96 & (4) & 2 & & & & & \\
\hline
\end{tabular}

A et B : code prairie; P0, P1, P2 : respectivement pas d'apport de phosphore, apport depuis un an (1991) ou deux ans (1992), apport depuis sept ans (1991) ou huit ans (1992); N0 et N1: respectivement pas d'apport et apport annuel d'azote ; l'écart type des IN et IP entre les différentes dates de mesure est indiqué entre parenthèses.

tillons des quatre répétitions ont été broyés et mélangés pour analyse des teneurs en azote (méthode Kjeldahl), en phosphore et en potassium.

\section{Traitement des données}

Pour plusieurs graminées cultivées, Lemaire et Salette (1984) ont montré l'existence d'une courbe critique reliant la teneur en azote à la biomasse aérienne, $\mathrm{N}=$ 4,8 $\mathrm{MS}^{-0,32}$ ( $\mathrm{N}$ en g $100 \mathrm{~g}^{-1}$, MS en tha-1), au-delà de laquelle tout apport d'azote supplémentaire se traduit par une augmentation de teneur sans accroissement de biomasse. Les paramètres de cette relation sont similaires à ceux calculés pour une légumineuse comme la luzerne qui en l'absence de stress (eau, oxygène) a généralement un niveau de nutrition optimal par la seule fixation symbiotique (Lemaire et al, 1985). En outre, nous avons montré que cette relation est acceptable pour des prairies permanentes (Duru, 1992a). Le niveau de nutrition azatée (IN) est défini comme le rapport entre la teneur en azote mesurée et la teneur qu'aurait dû avoir la végétation si l'azote n'avait pas été limitant pour la croissance (Lemaire et al, 1989) :

$$
\mathrm{IN}=100^{\star} \mathrm{N} /\left(4,8 \mathrm{MS}^{-0,32}\right)
$$

Dans le cas d'un mélange de graminées et de légumineuses, le niveau de nutrition calculé au niveau de l'ensemble de la végétation sera vraisemblablement d'autant plus élevé que la part des légumineuses dans le mélange est grande.
Le niveau de nutrition phosphatée des communautés prairiales (IP) est calculé à partir des teneurs en azote et en phosphore, $P$ et $\mathrm{N}$ étant exprimés en $\mathrm{g}$ $100 \mathrm{~g}^{-1}$ de biomasse. Nous avons montré l'existence d'une courbe critique reliant la teneur en phosphore à celle en azote, $P=0,24{ }^{*} N^{-0,64}$ ( $N$ et $P$ en $g 100 g^{-1}$ ), au-delà de laquelle tout apport de phosphore supplémentaire se traduit par une augmentation de teneur sans accroissement de biomasse (Duru, 1992a). IP est défini comme le ratio entre la teneur mesurée en $\mathrm{P}$ à la teneur qu'aurait dû avoir la végétation si le phosphore n'avait pas été limitant pour la croissance :

$$
I P=\left(4,17 P * N^{0,64}\right) * 100
$$

Les niveaux de nutrition $\mathrm{P}$ et $\mathrm{N}$ ont été calculés pour chaque date de mesure de la biomasse. Ils sont considérés comme non limitants pour des valeurs de 100 dans le cas de l'azote et de 80 dans le cas du phosphore (Duru, 1992b).

Pour calibrer le modèle d'accumulation de biomasse, nous avons utilisé les données de la prairie $S$ pour laquelle il n'y a pas de déficience en éléments minéraux majeurs autres que l'azote. Cette prairie prise comme témoin est à base d'espèces de graminées qui, pour les plus abondantes (Dactylis glomerata, Lolium perenne), sont cultivées (tableau II). Pour la modélisation de la biomasse, le cumul des températures moyennes journalières est effectué en base 0 à partir de la date d'apport d'engrais azoté. La première décade de mars correspond au moment où la vitesse de croissance augmente sur ce site (Duru, 1987a), en 
Tableau II. Composition botanique des communautés prairiales pour les communautés n'ayant pas reçu d'apport d'azote ; groupes de composition botanique : 1 et 2 respectivement pour les prairies composées d'espèces caractéristiques d'habitats peu ou bien fertiles.

\begin{tabular}{|c|c|c|c|c|c|c|c|c|c|c|c|}
\hline Communautés & 1986 & 1991 & 1991 & 1991 & 1991 & 1991 & 1992 & 1992 & 1992 & 1992 & 1992 \\
\hline & $S$ & $B-P 2$ & $B-P 1$ & $B-P O$ & $A-P 2$ & A-PO & $B-P 2$ & $B-P 1$ & $B-P O$ & $A-P 2$ & $A-P O$ \\
\hline \multicolumn{12}{|l|}{ Dicotylédones } \\
\hline Chaerophyllum aureum & & 6 & & & 30 & 14 & & & & 34 & 7 \\
\hline Gallium verum & 5 & & & & & & & & & & \\
\hline Lathyrus pratensis & & 7 & & & & & 9 & & & & \\
\hline Lotus corniculatus & & & & 5 & & & & & 6 & & \\
\hline Plantago lanceolata & & & 5 & & & & & & & & \\
\hline Plantago major & 7 & & & & & & & & & & \\
\hline Ranunculus acris & 5 & & & & & 6 & & & & & \\
\hline Rhinantus minor & & & 6 & 6 & & & & & & & \\
\hline Sanguisorba minor & & & & 8 & & & & 5 & 7 & & \\
\hline Stachys officinalis & 7 & & & & & & & & 7 & & \\
\hline Taraxacum officinalis & 12 & & & & & & & & & & \\
\hline Trifolium campestre & & & 16 & & & & & 13 & & & \\
\hline Trifolium pratense & & & 5 & & 6 & & 13 & & & & \\
\hline Trifolium repens & 6 & & & & & & & & & & 7 \\
\hline \multicolumn{12}{|l|}{ Graminées } \\
\hline Agrostis capillaris & & 7 & 9 & 6 & & & & 16 & 13 & & 6 \\
\hline Anthoxanthum odoratum & & & & & & 6 & & & & & \\
\hline Arrhenatherum elatius & & 21 & & & & 8 & 8 & & & 8 & 10 \\
\hline Dactylis glomerata & 22 & 13 & & & 12 & 7 & 8 & & & 12 & \\
\hline Festuca rubra & & 6 & 31 & 39 & & & 7 & 28 & 36 & & 5 \\
\hline Holcus lanatus & & & & & 14 & & & & & 14 & \\
\hline Lolium perenne & 25 & & & & & & 20 & & & 7 & \\
\hline Poa trivialis & 8 & & & & & & & & & 13 & \\
\hline Trisetum flavescens & & 12 & & & 9 & 10 & 9 & & & 10 & 10 \\
\hline Groupe de composition botanique & & 2 & 1 & 1 & 2 & 2 & 2 & 1 & 1 & 2 & 2 \\
\hline$-\cdots-$ & & & . & & & - & - & - & $\ldots$ & - & \\
\hline
\end{tabular}

Ne sont indiquées que les valeurs pour lesquelles le nombre de contacts pour une espèce donnée est supérieur à $5 \%$.

relation avec le changement d'affectation des assimilats (Parsons et Robson, 1980).

Pour étudier les effets de la composition botanique, nous avons constitué, sur la base de ces caractéristiques, des groupes de communautés au moyen d'une classification ascendante hiérarchique en utilisant les données des 17 communautés prairiales étudiées en 1991 et en 1992. L'objectif est de constituer au moins deux groupes différents. En outre, deux autres groupes de communautés ont été constitués sur la base de leur niveau de nutrition en phosphore (niveaux de nutrition $P$ inférieurs ou supérieurs à 80 ). Ensuite, nous avons comparé les biomasses mesurées aux biomasses simulées à l'aide du modèle calibré ci-dessus. Les écarts à ce modèle ont été estimés par la racine de l'erreur quadratique moyenne (REQM $=\Sigma\left[(\text { simulé-mesuré })^{2} / n\right]^{1 / 2}$ et testés par une analyse de variance en considérant les communautés d'un même groupe de composition botanique ou de niveau de nutrition en $\mathrm{P}$ comme des répétitions.

\section{RESULTATS}

\section{Caractérisation des communautés prairiales}

Pour le premier dispositif, les indices de nutrition phosphatée sont supérieurs à 100 . Les indices de nutrition azotée varient de 64 à 96 selon l'année et l'apport d'azote : respectivement 96,77 et 94 pour les traitements $N 1,73,64$ et 80 pour les traitements NO.

Pour le deuxième dispositif, les compositions botaniques étant très voisines entre les communautés ne différant que par l'apport d'azote de l'année, seule celles des traitements No sont indiquées dans le tableau II. La classification ascendante hiérarchique, établie pour l'ensemble 
de ces communautés, permet de définir deux groupes principaux à partir de leur composition botanique (tableau II). Un premier groupe ( $C B=$ 1) comprend les communautés $P 1$ et $P 0$ de la prairie $B$, le deuxième $(C B=2)$ correspond aux communautés $P 2$ des prairies $A$ et $B$, ainsi que les communautés $P 0$ de la prairie $A$. Au sein de chacun de ces groupes principaux, on peut établir deux sous-groupes. Pour le premier groupe, les sous-groupes sont constitués respectivement des communautés $\mathrm{P} 0$ et $\mathrm{P} 1$; pour le deuxième groupe, il s'agit des communautés $P 2$ de la prairie $B$ et $P 0$ de la prairie $A$, puis de la communauté P2 de la prairie A. Pour les communautés appartenant au premier groupe, les espèces les plus représentées sont Festuca rubra pour les graminées (30 à $40 \%$ du nombre total de contracts), Sanguisorba minor (sous-groupe 1) ou Trifolium campestre (sous-groupe 2) pour les dicotylédones (environ $10 \%$ des contacts). Pour les communautés appartenant au deuxième groupe, les espèces les plus représentées sont Dactylis glomerata, Trisetum flavescens, Arrhenatherum elatius et Holcus lanatus pour les graminées, Chaerophyllum aureum ou/et Lathyrus pratensis pour les dicotylédones. L'analyse ascendante hiérarchique réalisée séparément pour les graminées et les dicotylédones aboutit à la définition des mêmes groupes principaux.

On peut également définir deux classes de communautés bien distinctes à partir des indices de nutrition en phosphore (tableau I). Les indices inférieurs à 80 (nutrition $P$ limitante pour la croissance) correspondent aux communautés présentant un niveau de nutrition très déficient (communautés $B-P 0$ ) à déficient en phosphore (communautés A-P0 et B-P1 en 1991). Les autres communautés ont un niveau de nutrition $P$ non limitant (IP > 80). Les indices de nutrition azotée s'échelonnent de 78 à 102 pour les communautés ayant reçu un apport d'azote et de 63 à 95 pour les autres. Conformément à des observations antérieures (Duru, 1992a, b), à apport d'azote identique, les communautés ayant reçu un apport de phosphore (P1 ou P2) ont un niveau de nutrition azotée toujours supérieur à celui des communautés $\mathrm{PO}$.

Pour le deuxième dispositif, cette double classification nous permet de définir quatre groupes de communautés prairiales selon leur composition botanique et leur niveau de nutrition en phosphore. Pour le premier groupe de composition botanique, les niveaux de nutrition azotée moyens sont de 75,9 $\pm 7,9$ (IP limitants) et de $84 \pm 11$ (IP non limitants). Pour le deuxième groupe, ils sont respectivement de 76,5 $\pm 5,4$ et $89 \pm 11,2$. Lorsque le niveau de nutrition $P$ est limitant, les valeurs d'IP ne sont pas significativement différentes entre les deux groupes de communautés établis sur la base de leur composition botanique $(p=0,47)$.

\section{Accumulation de biomasse aérienne pour les communautés du premier dispositif : effet des niveaux de nutrition en $\mathbf{N}$}

Pour la prairie S, seul le niveau de nutrition azotée varie entre traitements et années. À 800 degrés-jours (dj), la biomasse aérienne (MS) varie d'environ 420 à $780 \mathrm{~g} \mathrm{~m}^{-2}$. On observe un ralentissement de l'accumulation de biomasse pour des sommes de températures comprises entre 500 et 700 degrés-jours. La biomasse accumulée en fonction du cumul des températures $\left(T\right.$ en $\left.{ }^{\circ} \mathrm{C}\right)$ est, une année donnée, toujours plus élevée pour les placettes ayant reçu un apport d'azote (fig 1). Pour les trois années et les deux traitements, les courbes de croissance s'ordonnent logiquement en fonction des niveaux de nutrition azotée correspondants. La vitesse d'accumulation de la biomasse aérienne ( $\mathrm{dMS} / \mathrm{dT}$ ) peut être considérée comme une fonction du niveau de nutrition azotée. Pour modéliser l'accumulation de biomasse, nous avons retenu un modèle du type $M S=a+T\left(b^{*} I N+c\right)$. Nous obtenons:

$$
\begin{aligned}
& \mathrm{MS}=-99+\mathrm{T}(0,0181 \mathrm{IN}-0,42) \\
& \left.s e=43,9, r^{2}=0,96, n=32\right)
\end{aligned}
$$

\section{Accumulation de biomasse aérienne pour les communautés du deuxième dispositif : effet de la composition botanique et du niveau de nutrition en phosphore}

Les courbes d'accumulation de biomasse exprimées en fonction du cumul de température sont d'allure linéaire. À $800 \mathrm{dj}$, la biomasse varie de 300 à $1050 \mathrm{~g} \mathrm{~m}^{-2}$ en 1991 et de 400 à 820 en 1992 (fig 2). À chaque date de mesure, les communautés du premier groupe de composition botanique ( $C B=1$ ) qui sont déficientes en phosphore (IP $=1$ ) ont une biomasse inférieure à celle des communautés du deuxième groupe $(C B=2)$ sans déficience en phosphore ( $I P=2$ ). Les biomasses des autres communautés $(C B=1$ et IP = 2 d'une part, $C B=2$ et IP $=1$ d'autre part) sont intermédiaires ou égales à celles des deux 
autres groupes. Ces observations ne nous permettent cependant pas de formuler des conclusions à valeur générale dans la mesure où les niveaux de nutrition azotée ne sont pas les mêmes entre ces groupes de communautés (tableau I). Pour les deux années, on observe que les différences de biomasse entre types de communautés s'accroissent au cours de la période d'étude. Ces résultats nous permettent de formuler l'hypothèse que la vitesse d'accumulation de la biomasse dépend des niveaux de nutrition en phosphore et en azote ainsi que de la composition botanique des communautés.

\section{Simulation de l'accumulation de biomasse pour les communautés du deuxième dispositif}

La comparaison des biomasses simulées par l'équation [3] et mesurées montre une bonne
Fig 1. Accumulation de biomasse en fonction du cumul des températures moyennes journalières. Année $1986(\mathrm{O}$, ๑), $1987(\square, \square)$ et $1988(\Delta, \Delta)$, symboles pleins pour les traitements sans apports d'azote, symboles vides pour $N=120$ $\mathrm{kg} / \mathrm{ha} ; \mathrm{l}$ ecart type de la moyenne entre les quatre répétitions.
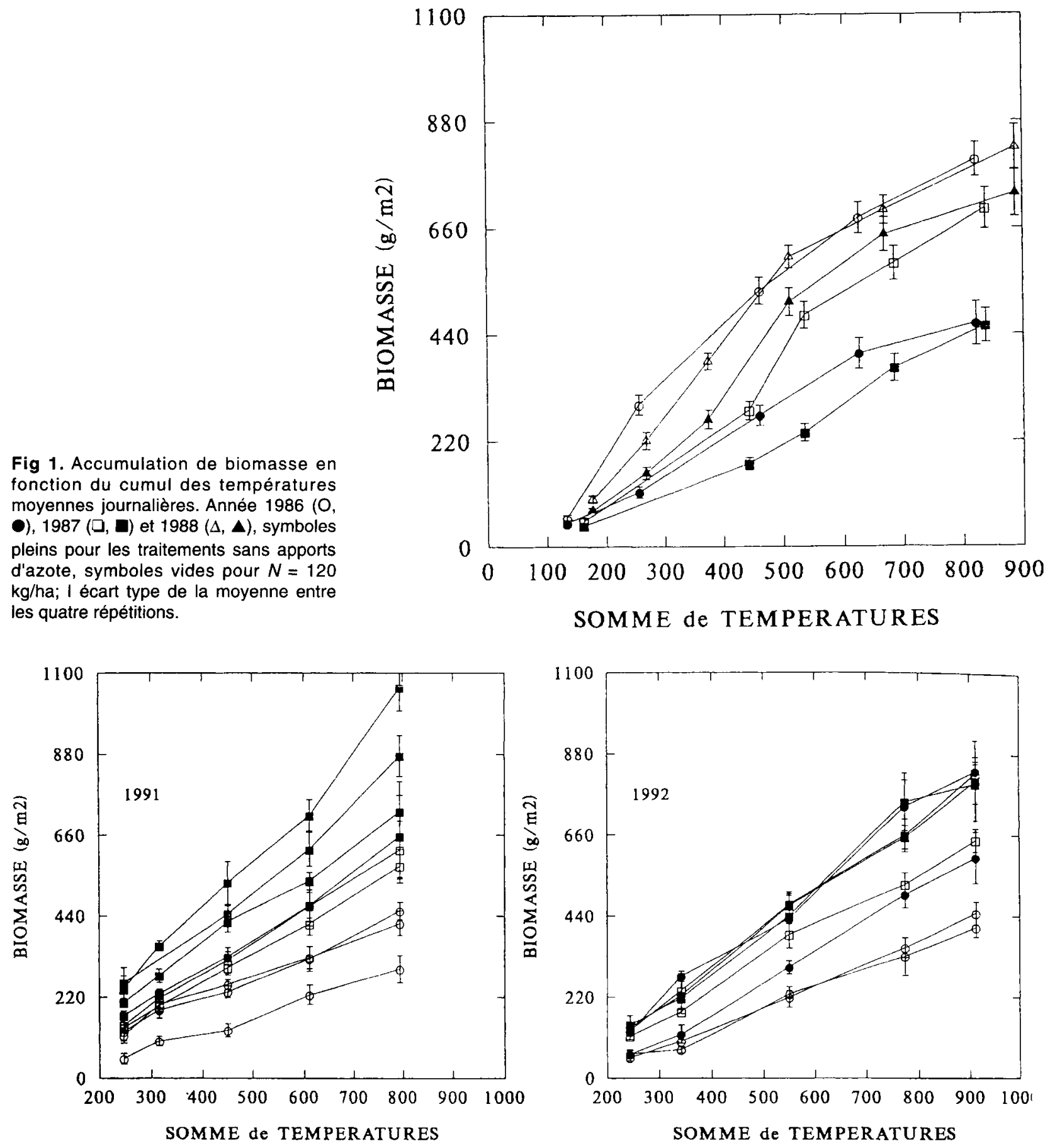

Fig 2. Accumulation de biomasse en fonction du cumul des températures moyennes journalières pour les communautés de composition botanique de type 2 sans ( $\square$ ) et avec (D) déficience en phosphore, et pour les communautés de composition botanique de type 1 sans $(\bullet)$ et avec $(O)$ déficience en phosphore; I écart type de la moyenne entre les quatre répétitions. 
adéquation pour les communautés du groupe 2 ( $C B=2$ ), pour les biomasses les plus basses (communautés sans déficience en phosphore) ou pour les biomasses les plus élevées (communautés avec déficience en phosphore) (fig 3). Les valeurs simulées sont toujours supérieures aux valeurs mesurées pour les communautés du groupe $1(C B=1)$. La différence est importante surtout pour celles qui sont une déficience en phosphore.

Le rapport entre la biomasse mesurée et la biomasse simulée (moyenne des différentes dates et des différentes communautés d'un même groupe) est proche de 1 pour les communautés du groupe 2 sans déficience en phosphore (tableau III). Pour les trois autres groupes de communautés, on observe que ce rapport décroît quand le niveau de nutrition en phosphore diminue et quand on passe d'une composition botanique de type 2 à 1 . Il en résulte que la racine de l'erreur quadratique moyenne augmente (tableau III). Ceci signifie que le modèle choisi n'est bien adapté que pour les communautés dont la nutrition en phosphore est non limitante et dont la composition botanique est de type 2 . Une analyse de variance à deux facteurs du rapport de biomasse (mesurée/simulée) montre un effet significatif de la composition botanique et du niveau de nutrition en phosphore $(p<0,001)$, mais pas de l'interaction entre les deux variables. Les conclusions issues de l'analyse de variance sont les mêmes que l'on considère toutes les données ou seulement les valeurs moyennes obtenues au cours d'une repousse.

Tableau III. Rapport entre les biomasses $\left(\mathrm{g} \mathrm{m}^{-2}\right)$ mesurée et simulée (moyenne et écart type) et racine de l'erreur quadratique moyenne pour les différents types de communautés.

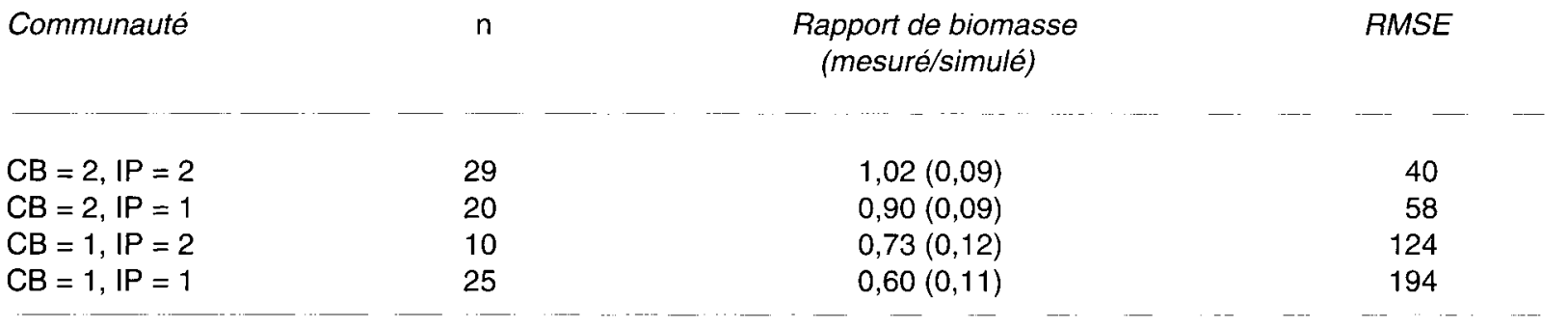

$\mathrm{CB}$ : classes de composition botanique (1 et 2 respectivement pour les prairies composées d'espèces caractéristiques d'habitats peu ou bien fertiles), IP : niveau de nutrition en phosphore (1 pour les niveaux déficients, 2 pour les niveaux non limitants).

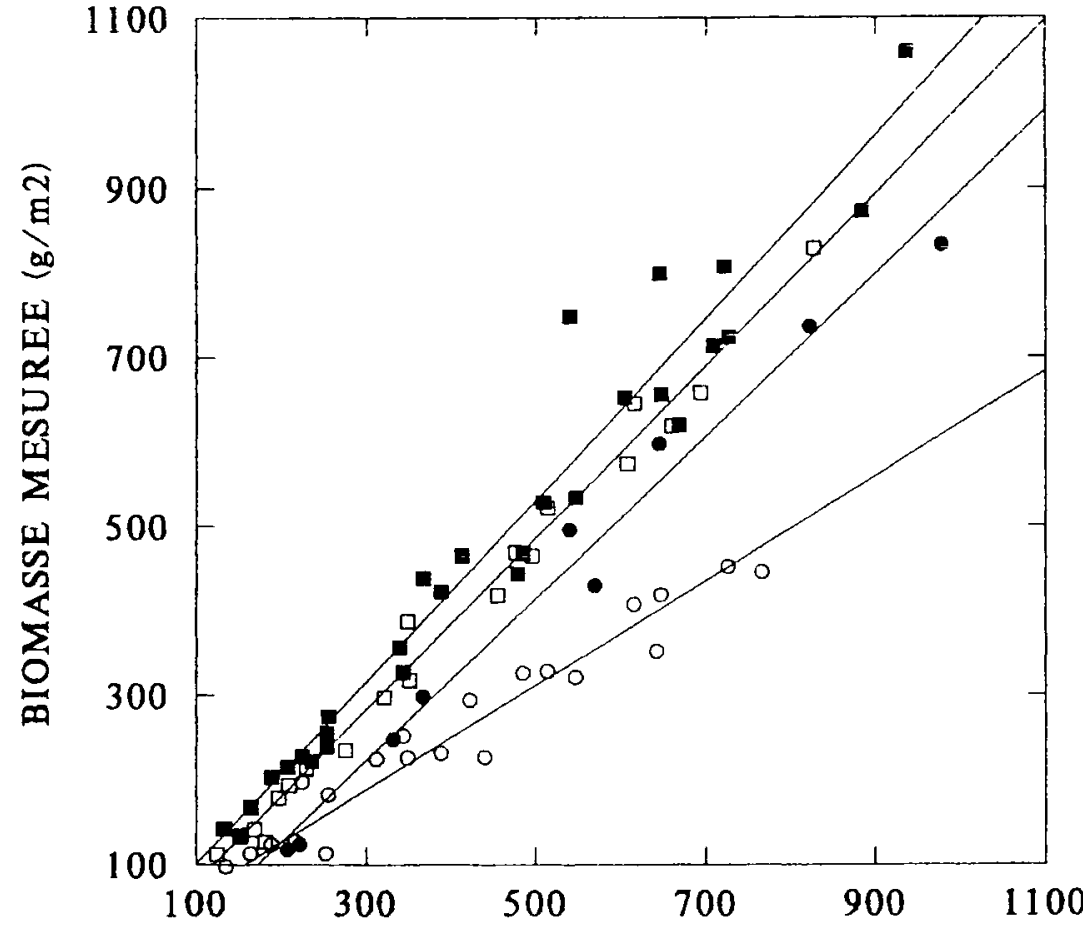

BIOMASSE SIMULEE $(\mathrm{g} / \mathrm{m} 2)$
Fig 3. Comparaison entre biomasses mesurée (Msmes) et simulée (MSsim) pour les communautés de composition botanique de type 2 sans ( $\square$ ) et avec ( $\square$ ) déficience en phosphore, et pour les communautés de composition botanique de type 1 sans ( et avec $(O)$ déficience en phosphore ; tracés des droites d'ajustement entre biomasses mesurées et simulées pour chacun des groupes de communautés. 


\section{DISCUSSION}

\section{Potentiel de croissance et niveau de nutrition azotée}

Une des difficultés signalées pour l'étude de l'accumulation de biomasse des prairies permanentes tient à ce qu'elles sont constituées d'un nombre variable d'espèces dont les phénologies ne sont pas synchrones. La biomasse peut donc être estimée alors que l'arrêt d'émission des feuilles, ou leur sénescence, a déjà commencé pour seulement un sous-ensemble des espèces constituant la communauté. Le risque est donc de sous-estimer la biomasse produite pour certaines communautés. C'est une des raisons possibles pour laquelle la vitesse d'accroissement de biomasse pour la prairie $S$ tend à diminuer au cours de la pousse (fig 1). En effet, cette prairie comprend du Taraxacum officinalis qui est l'une des espèces à floraison très précoce (Calvière et Duru, 1996), ainsi que deux graminées dominantes à floraison précoce. En outre, une différence importante entre la prairie $S$ et les communautés du groupe 2 est la présence de grandes ombellifères (Chaerophyllum aureum) dans le second cas. Dans ces situations, Peeters (1988) a observé des biomasses récoltables supérieures à celles de prairies à base de graminées. Ce résultats peut expliquer des rapports entre la biomasse mesurée et la biomasse simulée parfois supérieurs à 1 pour les communautés B-P2N1 lors des dernières mesures de biomasse (fig 3). Pour ces raisons, la biomasse produite par la prairie $S$ est vraisemblablement plus sous-estimée que ne l'est celle des prairies $A$ et $B$, du moins à la fin de la période d'étude. Étant donné l'impact limité de ces particularités, on peut conclure que les compositions botaniques des communautés du groupe 2 ainsi que celle de la prairie $S$ permettent d'atteindre la croissance potentielle permise par le climat, bien que les espèces dominantes ne soient pas toutes les mêmes. La généralisation des résultats demanderait de connaître plus précisément les caractéristiques phénologiques et morphogénétiques des espèces. C'est aussi une étape nécessaire pour considérer un modèle de croissance moins empirique.

Les niveaux de nutrition azotée étudiés s'échelonnent de 63 à plus de 100 (tableau I), ce qui représente une gamme de variation assez large relativement aux valeurs extrêmes observées pour des sols minéralisant peu (45 environ d'après Bélanger et al, 1992 ; Duru et al, 1995). Pour un niveau de nutrition azotée non limitant ( $I N=100)$, la vitesse d'accumulation de biomasse est de 13,9 $\mathrm{kg} \mathrm{ha}^{-1} \mathrm{dj}^{-1}$ (équation [3]). Ces valeurs sont peu inférieures à celles obtenues pour des couverts de graminées fourragères (Lemaire, 1985). Ces résultats confirment aussi les conclusions de Charles et al (1979) qui montrent que la biomasse produite est peu différente entre prairies temporaires et permanentes à base de graminées, les différences pouvant résulter de décalages de phénologie comme indiqué ci-dessus.

Lorsque le niveau de nutrition azotée est réduit de $40 \%(I N=60)$, la vitesse de croissance est réduite de $52 \%$ d'après l'équation [3]. Cette réduction est supérieure à celle observée pour des graminées fourragères (environ $36 \%$ d'après Bélanger et al, 1992). Dans ce dernier cas, il s'agit de repousses végétatives ou de mesures antérieures à la montaison des graminées dans le cas de pousses reproductrices. Pour les communautés prairiales étudiées ici, les biomasses sont mesurées jusqu'à l'épiaison des principales graminées. Une déficience en azote pouvant limiter le nombre de talles qui montent (Langer, 1959), elle peut amplifier les différences de biomasses entre traitements azotés comparativement à des pousses végétatives.

\section{Effet de la composition botanique sur la production de biomasse récoltable}

La typologie réalisée a permis de séparer de manière simplifiée les espèces en deux groupes qui reflètent des différences d'habitat dans la zone d'étude. Les espèces dominantes sont rarement communes entre les deux groupes de communautés (tableau II). Celles du groupe 2 se distinguent de celles du groupe 1 par le fait qu'elles sont caractéristiques des milieux les plus fertiles et les plus intensément utilisés (Balent, 1991). Ces regroupements d'espèces en fonction des caractéristiques d'habitats ont une portée plus large que la zone d'étude, du moins pour les graminées (Poorter et Remkes, 1990). La comparaison des biomasses simulées et observées pour les différents groupes de communautés a montré que dans les conditions de l'étude, les différences de composition botanique constituent la deuxième source de variation de la biomasse récoltable. À même niveau de nutrition en $\mathrm{P}$ et $\mathrm{N}$, la différence de biomasse récoltable a été estimée à $30 \%$ lorsque l'on compare les deux groupes de composition botanique (tableau III). Les différences de croissance entre groupes d'espèces lorsque les 
niveaux de nutrition minérale sont similaires peuvent provenir de différences d'activité photosynthétique ou de répartition des assimilats entre les parties aériennes et souterraines.

En situation de compétition intraspécifique, les études (qui ne concernent que quelques graminées bien typées) montrent que les espèces originaires d'habitat fertile (Arrhenatherum elatius, Dactylis glomerata, par exemple) ont une vitesse de croissance plus rapide que celles d'habitat pauvre (Festuca rubra, Briza media), que les disponibilités en éléments minéraux, azote en particulier, soient faibles ou élevés (Berendse et al, 1992 ; Van der Werf et al, 1993). Nos résultats issus de comparaisons de communautés sont donc cohérents avec ceux concernant des populations de graminées. Deux interprétations sont avancées. D'une part les espèces caractéristiques des habitats les plus fertiles ont une plus grande activité photosynthétique par unité de surface, comme le montre la comparaison entre Dactylis glomerata et Briza media (Van der Werf et al, 1993). D'autre part, elles affectent une plus grande proportion d'assimilats aux tiges ou pseudo-tiges du moins dans les stades précoces (comparaison de Dactylis glomerata et de Brachypodium pinnatum Ryser et Lambers, 1995), sans pour autant avoir une surface foliaire plus faible du fait d'une plus grande surface spécifique des feuilles. Autrement dit, les espèces d'habitat les plus fertiles investissent une plus grande part de leurs assimilats dans des tissus de soutien, ce qui explique leur plus grande taille et les rend plus compétitives pour la lumière (Elberse et Berendse, 1993). Ces études concernent la comparaison de graminées avant l'élongation des tiges. Nous n'avons pas d'informations sur les différences susceptibles d'apparaître après ce stade qui pourraient dépendre de la stratégie de reproduction (végétative ou reproductrice) des espèces.

Ces résultats obtenus en monocultures permettent d'expliquer qu'en conditions naturelles les communautés situées dans les habitats fertiles produisent le plus. D'une part les disponibilités en éléments minéraux sont élevées, voire non limitantes, pour assurer une croissance potentielle, d'autre part ces communautés ont intrinsèquement une plus grande vitesse de croissance.

\section{Effet du phosphore sur la production de biomasse récoltable}

La gamme des niveaux de nutrition en phosphore représente bien la variabilité observée pour un grand nombre de prairies étudiées en France (Salette et Huché, 1991). D'après les comparaisons entre biomasses simulées et récoltées, la vitesse d'accumulation de biomasse est réduite d'environ $12 \%$ lorsque l'on passe d'une situation non limitante à une déficience sévère. De nombreuses études comparent la croissance de plusieurs espèces prairiales (graminées, dicotylédones) dans des conditions de nutrition $P$ variées. Ces études, conduites le plus souvent en pot, font apparaître dans tous les cas des réductions de biomasse jusqu'à $30 \%$ avec les carences en $\mathrm{P}$, tant pour des graminées rencontrées sur des prairies de fertilité moyenne à bonne (Dactylis glomerata, Holcus lanatus, Anthoxanthum odorantum : Wild et al, 1974 ; Pedgel, 1987) que pour d'autres espèces connues pour leurs vitesses de croissance faibles ou élevées (Rorison, 1968 ; Amarasinghe et Pemadasa, 1982). Au champ, nous avons montré qu'une déficience en $P$ sévère réduit la surface foliaire d'environ $20 \%$ (Duru, 1987b). En condition hydroponique, l'activité photosynthétique est divisée jusque par 2, voire 3 , surtout en condition de fort éclairement (Sawada et al, 1983). En outre, une déficience en $P$ peut avoir un effet défavorable sur l'induction florale des graminées (Ikegaya et al, 1980).

Les estimations de réductions de biomasse au champ dues au phosphore sont donc plutôt inférieures aux valeurs mentionnées dans la littérature. Mais dans ce cas, il s'agit de déficience vraisemblablement plus sévères dans le cas de cultures hydroponiques. En outre, les effets possibles d'une déficience en $P$ sur le niveau de nutrition azotée de la culture (Duru, 1992a) dans le cas d'étude en pot ne sont pas pris en compte.

\section{Esquisse des effets à court et moyen terme de la fertilisation sur la biomasse récoltable}

Nous proposons ci-dessous de résumer les changements d'accumulation de biomasse au cours d'une pousse de printemps susceptibles de se produire à la suite d'un apport de fertilisant. Les quantités de biomasse indiquées sont des ordres de grandeur de ce qu'il est possible d'obtenir à l'épiaison des graminées, soit à environ 800 dj dans les conditions de notre étude. Si l'on considère comme situation d'origine une communauté déficiente en phosphore dont les espèces dominantes sont caractéristiques d'habitat peu fertile, la biomasse récoltable est faible (250 à $350 \mathrm{~g} \mathrm{~m}^{-2}$ ). Un apport d'azote en fin d'hiver per- 
met cependant d'augmenter la biomasse récoltable jusqu'à environ $450 \mathrm{~g} \mathrm{~m}^{-2}$. L'apport de phosphore répété durant 2 à 3 années se traduit par l'augmentation de la biomasse récoltable en relation avec l'augmentation du niveau de nutrition en phosphore et très souvent une amélioration du niveau de nutrition azotée (Duru, 1992a), sans que pour autant la composition botanique ait eu le temps de changer significativement (Balent et al, 1993 ; Duru et al, 1994). Les biomasses récoltables sont alors comprises entre 450 et $700 \mathrm{~g} \mathrm{~m}^{-2}$ selon les apports d'azote. À la suite d'une fertilisation durant un plus grand nombre d'années, la composition botanique change de manière importante du fait de la compétition interspécifique (Balent et al, 1993). Dans nos conditions d'étude, aucune des espèces dominantes n'est alors commune avec la communauté d'origine (tableau II). L'accumulation de biomasse aérienne des nouvelles espèces dominantes est plus rapide vraisemblablement du fait d'une plus grande activité photosynthétique et d'une plus grande proportion d'assimilats affectés vers les tissus de soutien, ce qui contribue à augmenter la biomasse produite, qui peut atteindre 700 à $950 \mathrm{~g} \mathrm{~m}^{-2}$ selon les apports d'azote.

\section{CONCLUSION}

La biomasse aérienne des communautés prairiales est très variable en relation avec leur composition botanique et leur état nutritionnel en azote et en phosphore. Dans nos conditions d'étude, les biomasses aériennes au stade épiaison des principales graminées ont varié de 300 à $1050 \mathrm{~g} \mathrm{~m}^{-2}$. L'étude d'une communauté témoin à base de graminées a montré que la vitesse de croissance par degré-jour était peu différente de celle de populations de graminées cultivées. La comparaison de cette communauté à d'autres très différentes quant à leur composition botanique et leur niveaux de nutrition en azote et en phosphore a permis de montrer que les effets sur la biomasse récoltable sont dus par ordre croissant aux déficiences en phosphore (réduction de la vitesse de croissance d'environ $12 \%$ lorsqu'on passe d'un niveau de nutrition $P$ non limitant à limitant), à la composition botanique (diminution de près de $30 \%$ lorsqu'on passe d'une communauté composée d'espèces caractéristiques d'habitats fertile à peu fertile), et aux déficiences en azote (diminution d'environ $50 \%$ lorsque le niveau de nutrition azotée est diminué de moitié). L'effet d'une déficience en azote apparaît plus important que dans le cas de populations de gra- minées. Les communautés pour lesquelles la biomasse est moindre, pour un même état nutritionnel de la végétation, sont composées d'espèces caractéristiques d'habitats peu fertiles. Les gammes de variation des états nutritionnels en azote et en phosphore, ainsi que de compositions botaniques pris en compte dans cette étude sont importantes et les valeurs extrêmes correspondent assez bien à ce qui est observé dans d'autres régions que la zone étudiée. La généralisation des résultats demanderait de connaître plus précisément les caractéristiques phénologiques et morphogénétiques des espèces. C'est aussi une étape nécessaire pour considérer un modèle de croissance moins empirique que celui utilisé ici.

\section{REMERCIEMENTS}

Les auteurs tiennent à remercier $L$ Sos pour son aide technique.

\section{RÉFÉRENCES}

Amarasinghe L, Permadasa MA (1982) The ecology of a mountains grassland in Sri Lanka. IV. Vegetative growth of four major grasses. $J$ Ecol 70, 721-730

Balent G (1991) Construction of a reference frame for studying changes in species composition in pastures: the example of an old-field succession. Options méditerranéennes, CIHEAM, Série A Séminaires, 15, 73-81

Balent G, Duru M (1984) Influence des modes d'exploitation sur les caractéristiques et l'évolution des surfaces pastorales : cas des Pyrénées centrales. agronomie 4, 113-124

Balent G, Duru M, Madga D (1993) Pratiques de gestion et dynamique de la végétation des prairies permanentes. Une méthode de diagnostic agroécologique, une application aux prairies de l'Aubrac et de la vallée de l'Aveyron. Études et recherches sur les systèmes agraires et le développement 27, 283-302

Bélanger G, Gastal F, Lemaire G (1982) Growth analysis of a tall fescue sward fertilized with different rates of nitrogen. Crop Sci 32, 1371-1376

Berendse F, Elberse WT, Geerts RH (1992) Competition and nitrogen loss from plants in grassland ecosystems. Ecology 73, 46-53

Calvière I, Duru M (1996) Leaf appearance and senescence patterns of some pasture species. Grass Forage Sci (sous presse)

Charles AM, Jones JL, Thornton MS, Thomas TA (1979) Comparison of ryegrass with some common unsown grasses sown separately and in mixture. Proceeding of a Symposium of the British Grassland Society 10, 25-30 
Charpenteau JL, Duru M (1983) Simulation of some strategies to reduce the effect on climatic variability on farming. The case of Pyrenees Mountains. Agric Syst 11, 105-125

Daget P, Poissonnet P (1971) Une méthode d'analyse phytosociologique des prairies. Critères d'application. Ann Agron 22, 45-41

Duru M (1987a) Croissance hivernale et printanière de prairies permanentes pâturées en montagne. I. Écophysiologie du dactyle. agronomie 7, 41-50

Duru M (1987b) Croissance hivernale et printanière de prairies permanentes pâturées en montagne. II. Variation de la croissance et de la composition chimique. agronomie $7,51-59$

Duru M (1992a) Diagnostic de la nutrition minérale de prairies permanentes au printemps. I. Établissement de références. agronomie 12, 219-233

Duru M (1992b) Diagnostic de la nutrition minérale de prairies permanentes au printemps. II. Validation de références. agronomie 12, 345-357

Duru M, Balent G, Langlet A (1994) Mineral nutrition status and botanical composition of pastures. I. Effect on herbage accumulation. Eur J Agron 3, 4351

Duru M, Ducrocq $H$, Tirilly $V$ (1995) Modeling growth of cocksfoot (Dactylis glomerata $\mathrm{L}$ ) and tall fescue (Festuca arundinacea Schreb) at the end of spring in relation to herbage nitrogen status. $J$ Plant Nutr $18,2033-2047$

Elberse WT, Berendse F (1993) A comparative study of the growth and morphology of height grass species from habitats with different nutrient availabilities. Funct Ecol 7, 223-229

Fleury $P$ (1994) Le diagnostic agronomique des végétations prairiales et son utilisation dans la gestion des exploitations agricoles. Méthode et applications dans les Alpes du Nord. Thèse de docteur en sciences agronomiques, INP de Lorraine

Gillet M, Gosse G, Lemaire G (1984) Essai d'élaboration d'un schéma global de la croissance des graminées fourragères. agronomie 4, 75-85

Gosse G, Varlet-Grancher C, Bonhomme R, Chartier M, Allirand JM, Lemaire G (1986) Production maximale de matière sèche et rayonnement solaire intercepté par un couvert végétal. agronomie 6, 4756

Grime JP, Hunt R (1975) Relative growth rate: its range and adaptative significance in a local flora. $J$ Ecol 63, 393-422

Ikegaya F, Sato S, Kawalata A (1980) Control flowering in cocksfoot (Dactylis glomerata L). Influence of fertilizer application on response to floral induction treatment in the aged plant. Bull Nat Grass/ Res Inst $17,68-75$

Kays S, Harper JL (1974) The regulation of plant and tiller density in a grass sward. J Ecol 62, 97-105

Langer RHM (1959) Growth and nutrition of timothy (Phleum pratense L). V. Growth and flowering at different levels of nitrogen. Ann App/ Biol 4, 740-751
Lemaire G (1985) Cinétique de la croissance d'un peuplement de fétuque élevée (Festuca arundinacea Schreb) pendant l'hiver et le printemps. Effet des facteurs climatiques. Thèse de doctorat d'État, université de Caen, $96 p$

Lemaire G, Salette J (1984) Relation entre dynamique de croissance et dynamique de prélèvement d'azote pour un peuplement de graminées fourragères. I. Étude de l'effet du milieu. agronomie 4, 423-430

Lemaire G, Cruz P, Gosse G, Chartier M (1985) Étude des relations entre la dynamique de prélèvement d'azote et la dynamique de croissance en matière sèche d'un peuplement de luzerne. agronomie 5, 685-692

Lemaire G, Gastal F, Salette J (1989) Analysis of the effect of $\mathrm{N}$ nutrition on dry matter yeild of a sward by reference to potential yield and optimum $\mathrm{N}$ content. XVI International Grassland Congress, Nice, France, 179-180

Menzi H, Blum H, Nosberger J (1991) Relationship between climatic factors and the dry matter production of swards of different composition at two altitudes. Grass Forage Sci 46, 223-230

Monteith JL (1972) Solar radiation and productivity in tropical ecosystem. J Appl Ecol 9, 747-766

Parsons AJ, Robson MJ (1980) Seasonal changes in the physiology of $\mathrm{S} 24$ perennial ryegrass (Lolium perenne $L$ ). 1. Response of leaf extension to temperature during the transition from vegetative to reproductive growth. Ann Bot 46, 435-444

Pedgel DM (1987) Soil fertility and the composition of semi-natural grasslands. In: Disturbance in Grasslands (J Van Andel et al, eds), W Junk, Dordrecht, 51-66

Peeters A (1988) Techniques d'exploitation, végétation et qualité alimentaire de l'herbe : étude de leurs relations triangulaires dans les systèmes herbagers. Thèse de docteur es sciences, université de Louvain, $287 \mathrm{p}$

Poorter H, Remkes C (1990) Leaf area ratio and net assimilation rate of 24 wild species differing in relative growth rate. Oecologia 83, 553-559

Poorter H, Remkes C, Lambers H (1990) Carbon and nitrogen economy of 24 wild species differing in relative growth rate. Plant Physiology 94, 621-627

Rorison IH (1968) The response to phosphorous of some ecologically distinct plant species. I. Growth rates and phosphorus absorption. New Phytol 67, 913-923

Ryser P, Lambers H (1995) Root and leaf attributes accounting for the performance of fast-growing grasses at different nutrient supply. Plant Soil 170, 251-265

Salette J (1982) The role of fertilizers in improving herbage quality and optimization of its utilization. Proceeding of the 12th Int Potash Symp Berne. Inst Potash Institute, 117-144

Salette J, Huché L (1991) Diagnostic de l'état de nutrition minérale d'une prairie par l'analyse minérale du 
végétal : principes, mise en œuvre, exemples. Fourrages 125, 3-18

Sawada S, Igarashi T, Miyachi S (1983) Effects of phosphate nutrition on photosynthesis starch and total phosphorus levels in single rooted leaf of dwarf bean. Photosynthetica 17, 484-490

Van Der Werf A, Visser AJ, Schieving F, Lambers $H$ (1993) Evidence for optimal partitionning of biomass and nitrogen at a range of nitrogen availabilities for a fast-growing and slow-growing species. Functional Ecology 7, 63-74

Wild A, Skarlou V, Clements CR, Snaydon RW (1974) Comparison of potassium uptake of four plants species grown in sand and in flowing solution culture. J Appl Ecol 11, 801-812 